\title{
Effect of cardiorespiratory and strength exercises on disease activity in patients with inflammatory rheumatic diseases: a systematic review and meta- analysis
}

\author{
Silje Halvorsen Sveaas, ${ }^{1,2}$ Geir Smedslund, ${ }^{1,3}$ Kåre Birger Hagen, ${ }^{1,2}$ Hanne Dagfinrud ${ }^{1,2}$
}

- Additional material is published online only. To view, please visit the journal online (http://dx.doi.org/10.1136/ bjsports-2016-097149).

${ }^{1}$ Department of Rheumatology, National Advisory Unit on Rehabilitation in Rheumatology, Diakonhjemmet Sykehus, Oslo, Norway

${ }^{2}$ Department of Health Sciences, Institute of Health and Society, University of Oslo, Oslo, Norway ${ }^{3}$ The Norwegian Knowledge Center for the Health Services, The Norwegian Institute of Public Health, Oslo, Norway

\section{Correspondence to}

Silje Halvorsen Sveaas, Department of Rheumatology, National Advisory Unit on Rehabilitation in Rheumatology, P.O. box 23 Vinderen, Oslo 0319, Norway:

s.h.sveaas@medisin.uio.no

Accepted 12 March 2017 Published Online First 28 April 2017
ABSTRACT

Objective To investigate the effects of cardiorespiratory and strength exercises on disease activity for patients with inflammatory rheumatic diseases (IRDs).

Design A systematic review with meta-analysis registered at PROSPERO (CRD42015020004).

Participants Patients with IRDs.

Data sources The databases MEDLINE, AMED, Embase and CINAHL were searched from inception up to April 2016.

Eligibility criteria for selecting studies Trials were included if they were randomised controlled trials of adults with IRDs, comparing the effect of cardiorespiratory and strength exercises with usual care on disease activity and followed the American College of Sports Medicine's exercise recommendations. The primary outcome was disease activity in terms of inflammation, joint damage and symptoms.

Data synthesis Data were pooled in a randomeffect model for all outcomes, and standardised mean differences (SMDs) were calculated. The quality of evidence was evaluated according to the Grading of Recommendations Assessment, Development and Evaluation approach.

Results Twenty-six trials with a total of 1286 participants were included. There was high to moderate quality evidence, for a small beneficial effect on disease activity scores $(0.19(95 \% \mathrm{Cl} 0.05$ to 0.33$), \mathrm{p}<0.01)$ and joint damage (SMD 0.27 (95\% Cl 0.07 to 0.46$)$, $\mathrm{p}<0.01)$. Furthermore, moderate quality evidence for a small beneficial effect on erythrocyte sedimentation rate (SMD 0.20 (95\% Cl 0.0 to 0.39), $p=0.04$ ) and for no effect on $\mathrm{C}$ reactive protein (SMD $-0.14(95 \% \mathrm{Cl}-0.37$ to 0.08$), p=0.21)$. Beneficial effects were also seen for symptoms.

Conclusions The results of this review suggest beneficial effects of exercises on inflammation, joint damage and symptoms in patients with IRDs.

\section{INTRODUCTION}

The term inflammatory rheumatic diseases (IRDs) covers a large group of heterogeneous disorders sharing common features and clinical presentations. ${ }^{1}$ The most prominent clinical feature of IRDs is pain, which may have a central as well as peripheral origin, caused by current systemic inflammation or by joint and tissue damage due to a previous inflammatory condition. ${ }^{2}$ Disease activity in IRDs is often expressed as an index of inflammation, joint damage and symptoms like pain, stiffness and fatigue. Additionally, the last decade has produced evidence that patients with IRDs also have an increased risk of cardiovascular diseases (CVDs). ${ }^{3}$ The mechanisms underlying the premature CVD events in IRDs are not fully elucidated, but it is clear that the systemic inflammation, and its interaction with traditional risk factors, plays an important role. ${ }^{3}$

The main treatment goals in IRDs are to reduce disease activity and maintain physical function, ${ }^{45}$ and exercises are considered an important part of the management. ${ }^{6}$ Exercise is defined as physical activity that is planned, structured and repetitive, with a final or intermediate objective to improve or maintain physical fitness. ${ }^{7}$ Due to the clinical features of IRDs, exercises to maintain range of motion (ROM) and pain-relieving exercises in hot pools have been recommended. ${ }^{6}$ Unfortunately, these types of exercises have no effect on cardiovascular health. ${ }^{8}$ Cardiorespiratory exercise is shown to have an anti-inflammatory effect in healthy people and patients with low-grade inflammatory diseases, ${ }^{9}{ }^{10}$ but it is more uncertain whether these effects also are seen in people with chronic inflammation. Yet, considering the protective effect of cardiorespiratory and strength exercises on CVD and inflammation in the general population, ${ }^{8}$ these modes of exercises should probably be more emphasised as therapeutic tools in the management of IRDs.

In observational studies, inflammation and clinical symptoms in patients with IRDs are shown to be inversely associated with cardiorespiratory fitness and physical activity level. ${ }^{11-13}$ Furthermore, theoretical models on how exercises can potentially counteract inflammation and improve clinical symptoms in patients with IRDs have been suggested. ${ }^{14-17}$ Despite this, the evidence of the anti-inflammatory effects of exercises in IRDs is ambiguous. In two systematic reviews of patients with rheumatoid arthritis, it was concluded that strength exercises had a beneficial effect on inflammation and joint tenderness and that cardiorespiratory exercise had a small beneficial effect on pain. ${ }^{18}{ }^{19}$ In contrast, Beavers et $a l^{17}$ and Ploeger et $a l^{16}$ concluded that there were limited and inconclusive data on the effect of exercises on disease activity in patients with IRDs.

In brief, theoretical models of mechanisms are suggested on how exercises can counteract inflammation and clinical symptoms in IRDs, ${ }^{14}{ }^{15}$ but the 
empirical evidence for such effects is still unclear. Therefore, the objective of this systematic review was to summarise the effects of cardiorespiratory and strength exercises compared with usual care on disease activity in patients with IRDs.

\section{METHODS}

The protocol for this systematic review was registered in the PROSPERO register of systematic reviews (CRD42015020004). The review group consisted of topic experts (SHS and HD), a methodologist (GS) and one review author who was both $(\mathrm{KBH})$.

\section{Data sources and searches}

The search strategy was prepared in collaboration with a healthcare librarian who performed the systematic literature searches.

A broad search for articles was undertaken that contained terms for all types of IRD combined with terms for exercise and training. No limitations were applied regarding outcomes. We limited the search to clinical trials and reports published in English (see online supplementary file S1 for details of the search strategy). The original search strategy was developed for the Ovid MEDLINE database and then adapted for AMED, Embase and CINAHL. The literature search was performed from inception up to April 2016. A manual search of reference lists of relevant articles was also undertaken.

\section{Eligibility criteria \\ Design}

Randomised controlled trials (RCTs) were considered eligible for inclusion. Studies were included if they investigated the effect of cardiorespiratory or strength exercises compared with usual care or other types of interventions not likely to influence cardiorespiratory or musculoskeletal fitness (such as ROM, stretching and relaxation). Trials were included if the effect was measured after completion of the exercise programme (ie, within a few days after completing the exercise programme. Follow-up trials were excluded). Furthermore, the length of the exercise interventions had to be $\geq 2$ weeks.

\section{Participants}

Trials were eligible if they had included adult patients $(\geq 18$ years) with confirmed IRDs (regardless of different diagnostic criteria). Trials with a mixture of different diagnoses of IRDs were also included. Trials were excluded if they had included patients with rheumatic diagnoses not involving systemic inflammation, such as for example osteoarthritis or fibromyalgia, as the aim was to assess the effect of exercise in patients with chronic inflammation.

\section{Intervention}

Interventions that met the American College of Sports Medicine's (ACSM) exercise recommendations for developing and maintaining cardiorespiratory fitness and or musculoskeletal strength were included. ${ }^{8}$

\section{Outcomes}

We had an explorative approach to the outcome measures, including all outcomes indicative of disease activity such as inflammatory markers, radiological joint damage, diagnosis-specific disease activity scores or clinical disease symptoms. The time point of interest was the first assessment after completion of the exercise programme (ie, within a few days after completion of the exercise programme). We also collected data on adverse events and side effects that were reported in the articles.

\section{Study selection}

Two review authors (SHS and HD) independently examined titles and abstracts against the eligibility criteria. All articles selected in this process were obtained in full text. All full-text articles were assessed independently by two review authors (SHS assessed all the full-text articles and then distributed them among the other review authors (HD, GS or $\mathrm{KBH})$ ). Disagreement among review authors regarding eligibility was discussed in the whole group of reviewers until consensus was reached.

\section{Data extraction}

Two reviewers (SHS and GS) extracted the data from the included studies. The data were discussed and agreement was reached for each case, and a unified data set was entered into Review Manager. Both change score with SDs and final value scores with SDs were included, but change scores were preferred if these were available. We preferred change scores as these were expected to give a more robust picture of the results as the sample sizes were small and data on inflammatory markers often are skewed. If results were given as median with range or IQR, the sample means and SDs were estimated using the formula described by Wan et al. ${ }^{20}$ Likewise, when only the mean and 95\% CI of change between the groups were given, the software Comprehensive Meta-Analysis V.3 was used to estimate the SD for each group. ${ }^{21}$

Authors of reports that were published after the year 2000 were contacted by mail in order to obtain data that were missing in the report, and a reminder was sent after 1 month if they had not replied.

\section{Quality assessment}

Methodological quality was assessed using the Cochrane Collaboration's risk of bias tool ${ }^{22}$ based on published material and a hand search of trial protocols at clinicaltrials.gov and other relevant webpages. The methodological quality of the trials was assessed independently by two review authors (SHS and GS). Thereafter, the data were checked for inconsistencies and entered into Review Manager. Discrepancies were discussed by the whole group of reviewers until consensus was reached.

Risk of bias assessments were made at the study level for (1) random sequence generation, (2) allocation concealment, (3) incomplete outcome data, (4) selective reporting, (5) other concerns about bias, and (6) blinding of outcome measures (this means that a study was rated with low risk of bias if it included objective measures of disease activity, such as $\mathrm{C}$ reactive protein, erythrocyte sedimentation rate, radiological joint damage (rated by a blinded assessor, and so on). We chose to not include blinding of participants and providers as a risk of bias item, because such blinding is difficult if not impossible in exercise trials.

For each outcome, we evaluated the quality of the evidence across trials according to the Grading of Recommendation Assessment, Development and Evaluation (GRADE) approach (www.gradeworkinggroup.org). The quality of evidence was divided into four categories: high, moderate, low and very low, according to how certain we were that the estimate of effect was true (eg, high quality indicated that we had high confidence). Since all the trials were RCTs, they were a priori rated as high-quality evidence. Factors that could reduce the quality of evidence were risk of bias, inconsistency of results, indirectness of evidence, imprecision and publication bias.

Publication bias was assessed by inspection of funnel plot asymmetry and Egger's test. In addition, the 'fail-safe N', the number of additional 'negative studies' that would be needed to increase the 


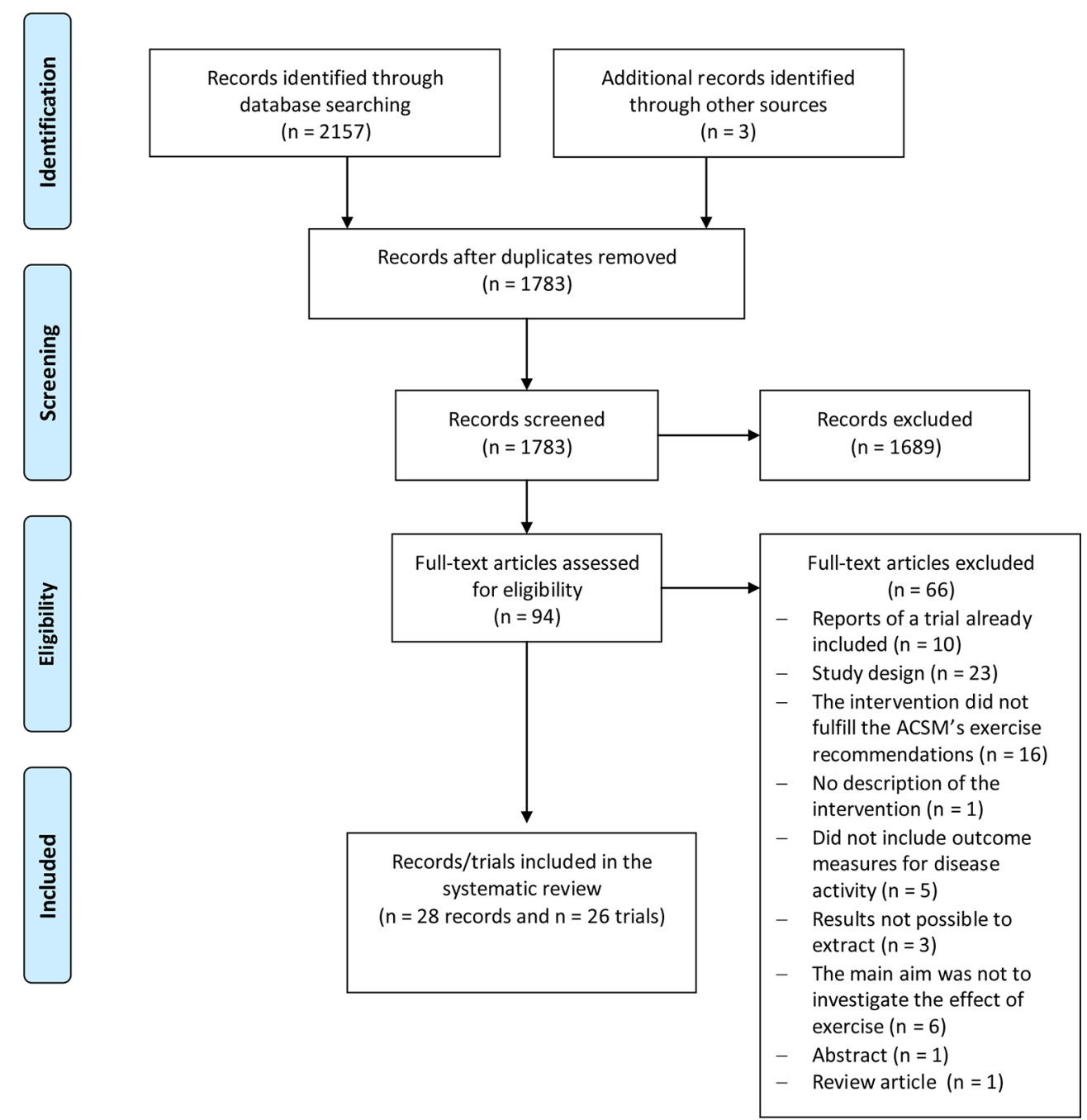

Figure 1 Flow diagram of the selection of trials. ACSM, American College of Sports Medicine.

$\mathrm{p}$ value for the meta-analysis above 0.05 , was calculated. Publication bias was only assessed for meta-analysis with $\geq 10$ studies, because if the number of studies is lower, the power of the test is too low to distinguish chance from real asymmetry. ${ }^{23}$

\section{Data synthesis and analysis}

To perform meta-analyses, the data were extracted by two of the review authors (SHS and GS). The two reviewers discussed the data and reached agreement in every case, and a single, unified data set was entered into Review Manager V.5.3 software. For continuous variables, the standardised mean difference (SMD) with 95\% CI was calculated. The SDs of change scores and final value scores were assessed before pooling the results to ensure similar precision. SMDs between 0.2 and 0.4 were considered as a small effect size, from 0.5 to 0.7 as a medium effect size, and $\geq 0.8$ as a large effect size. ${ }^{24}$ Due to expected clinical heterogeneity between the trials, it was decided a priori to use a random-effects model for all outcomes. Heterogeneity was tested with $\chi^{2}$ measured by inspection of the $\mathrm{I}^{2}$ values that describes the percentage of the variability in effect estimates that is due to heterogeneity rather than sampling error. ${ }^{25}$ An $\mathrm{I}^{2}$ value of $0 \%$ to $40 \%$ is considered negligible, $30 \%$ to $60 \%$ represents moderate heterogeneity, $50 \%$ to $90 \%$ represents substantial heterogeneity and $75 \%$ to $100 \%$ represents considerable heterogeneity. ${ }^{25} \mathrm{~A} p$ value of $\leq 0.05$ was considered statistically significant.
Subgroup analyses were done in Review Manager using $\chi^{2}$ heterogeneity statistics for all outcomes. For meta-analyses that included $>10$ studies, the subgroup analyses were also performed in Comprehensive Meta-Analysis software V.3 by meta-regression. $^{21}$ The following subgroup analyses were performed to explore whether the delivery (duration, supervision and mode) of the exercise programmes had an impact on the results:

1. exercise programmes with a duration of $\leq 12$ weeks versus $>12$ weeks

2. supervised versus unsupervised exercise programmes

3. cardiorespiratory exercise versus strength exercises versus a combination of strength and cardiorespiratory exercises.

\section{RESULTS}

\section{Study selection}

A total of 1783 records were identified by the searches. Of these, 94 were assessed in full text, and 26 trials ( 28 records) were included in the meta-analysis (figure 1). Excluded studies with associated reasons are shown in online supplementary file S2.

\section{Study characteristics}

The main characteristics of the trials are shown in table 1 (see online supplementary table S1 for description of the exercise interventions). 
Table 1 Characteristics of the included trials

\begin{tabular}{|c|c|c|c|}
\hline Trial & Patient population* & Duration and exercise mode & Type of exercise \\
\hline 1. Alemo Munters et $a F^{52}$, Sweden & 21 (PM/DM) & 12 weeks, cardiorespiratory & Cycling \\
\hline 2. Alexanderson et $a l^{26}$, Sweden & 13 (PM/DM) & 24 weeks, cardiorespiratory and strength & Walking, strength exercise programme \\
\hline 3. Baillet et $a \beta^{37}$, France & 48 (RA) & 4 weeks, cardiorespiratory and strength & Cycling, running, resisting pulley cord \\
\hline 4. Baslund et $a l^{\beta 6}$, Denmark & 18 (RA) & 8 weeks, cardiorespiratory & Cycling \\
\hline 5. Daltroy et $a P^{34}$, USA & 58 (IRDs) & 12 weeks, Cardiorespiratory & Cycling \\
\hline 6. de Jong et $a^{35}{ }^{38}$, The Netherlands & 281 (RA) & 2 years, cardiorespiratory and strength & Cycling, strength exercise programme \\
\hline 9. Häkkinen et $a{ }^{41}$, Finland & 39 (RA) & 6 months, strength & Strength exercise programme \\
\hline 10. Häkkinen et a $/^{42}{ }^{43}$, Finland & 62 (RA) & 2 years, strength & Strength exercise programme \\
\hline 11. Hansen et $a l^{31}$, Denmark & 75 (RA) & 2 years, cardiorespiratory & Cycling, swimming, walking, running \\
\hline 12. Harkcom et $a^{32}$, USA & 17 (RA) & 12 weeks, cardiorespiratory & Cycling \\
\hline 13. Hsieh et $a{ }^{49}$, Taiwan & 19 (AS) & 3 months, cardiorespiratory and strength & Aerobics, strength exercise programme \\
\hline 18. Melikoglu et $a{ }^{46}$, Turkey & 36 (RA) & 2 weeks, cardiorespiratory & Walking/running (treadmill) \\
\hline 19. Miossi et al${ }^{27}$, Brazil & 28 (SLE) & 3 months, cardiorespiratory and strength & Walking/running (treadmill), strength exercise programme \\
\hline 20. Niedermann et $\mathrm{al}^{28}$, Switzerland & 106 (AS) & 12 weeks, cardiorespiratory & Nordic walking \\
\hline 21. Sandstad et a $/{ }^{29}$, Norway & $15(R A / J I A)$ & 10 weeks, cardiorespiratory & Cycling \\
\hline 22. Sveaas et $\left.a\right|^{30}$, Norway & 24 (axial SpA) & 12 weeks, cardiorespiratory and strength & Walking/running (treadmill), strength exercise programme \\
\hline 23. Tench et $a l^{33}$, United Kingdom & 65 (SLE) & 12 weeks, cardiorespiratory & Mainly walking (cycling, swimming) \\
\hline 24. van den Ende et $\mathrm{al}^{47}$, The Netherlands & 50 (RA) & 12 weeks, cardiorespiratory & $\begin{array}{l}\text { Cycling, walking, knee bending, step-ups, high pace strength } \\
\text { exercises }\end{array}$ \\
\hline 25. Westby et $a{ }^{48}$, Canada & 21 (RA) & 12 months, cardiorespiratory and strength & Aerobic dance, strength exercise programme \\
\hline 26. Wiesinger et $a l^{53}$, Austria & 13 (PM/DM) & 6 months, cardiorespiratory & Cycling, step aerobics \\
\hline
\end{tabular}

*Number of patients included in the results.

AS, ankylosing spondylitis; DM, dermatomyositis; EG, exercise group; IRDs, inflammatory rheumatic diseases; JIA, juvenile idiopathic arthritis; PM, polymyositis; RA, rheumatoid arthritis; SLE, systemic lupus erythematosus; SpA, spondyloarthritis.

The trials were published between 1985 and 2015, of which 25 were RCTs, and one was a cross-over study where results from each group before crossing were included.

Unpublished data were provided by authors in five trials. ${ }^{26-30}$ Results from several exercise groups were combined into one exercise group in two of the trials. ${ }^{3132}$ We estimated the mean and SD from median and range for one outcome (diagnosis-specific disease activity score) in one trial, ${ }^{33}$ and SD was estimated from CI in two trials ${ }^{3435}$ and from SE in one trial ${ }^{36}$ using the Comprehensive Meta-Analysis software V.3. ${ }^{21}$

\section{Participants}

Altogether, the 26 trials provided results for 1286 participants. The most frequently included diagnosis was rheumatoid arthritis, ${ }^{29} 32$ 35-48 followed by axial spondyloarthritis, ${ }^{28} 3049-51$ polymyositis/dermatomyositis, ${ }^{26} 5253$ systemic lupus erythematosus $^{27} 33$ and one trial included patients with several inflammatory rheumatic diagnoses. ${ }^{34}$

\section{Description of the exercise interventions}

In $13(50 \%)$ of the trials, the exercise programme consisted of cardiorespiratory exercises, ${ }^{28} 2931-3436464750-53$ in five (19\%) trials muscle strength exercises ${ }^{40-45}$ and in eight (31\%) trials, the exercise consisted of a combination of cardiorespiratory and strength exercises. $^{26273037-394849}$ Of the 21 exercise programmes containing cardiorespiratory exercises, six (29\%) involved cycling, ${ }^{29} 32$ 34-36 38 52 five (24\%) involved walking, ${ }^{26} 28333950$ three (14\%) involved walking/running on a treadmill, ${ }^{27} 3046$ two (10\%) involved aerobics $^{48} 49$ and five (24\%) involved a combination of two or more exercise types (cycling, walking, running, swimming and aerobics). ${ }^{31} 37475153$ The exercise intensity was described in all trials. A vigorous intensity level (between $77 \%$ and $95 \%$ of maximum heart rate $)^{8}$ was described in four of the trials with cardiorespiratory exercise, ${ }^{2930353638}$ and in most of the trials, the intensity was low to moderate (50 to $\leq \% 77$ of maximum heart rate). In eight trials, range of motion exercises was included together with cardiorespiratory and/or strength exercises. ${ }^{28} 313739$ 48-51 There was a considerable variation between the trials with regard to the length of the exercise period. The most common length of the intervention was 12 weeks, $27283032-34394447495052$ but the length ranged from 2 weeks $^{46}$ to 2 years. ${ }^{42} 43$

\section{Risk of bias in the included trials}

A summary of the risk of bias in the 26 included trials is shown in online supplementary figure S1. Four trials were rated as low risk of bias for all items on the methodological quality list. ${ }^{29303550}$ Eleven RCTs were rated as unclear risk of selection bias as the randomisation process was not adequately described. ${ }^{37}$ 39-41 44 46-48 5153 Seventeen trials were rated as unclear risk of bias as they did not provide sufficient information to judge if the randomisation process was concealed. ${ }^{27} 28$ 31-34 36 39-4853 The majority of the trials had a low drop-out rate and was rated as low risk of bias for this item. Only six trials were rated as a low risk of selective reporting as the protocol was available and in accordance with the published article. ${ }^{27-30}$ 3850 The majority of the trials included at least one objective 

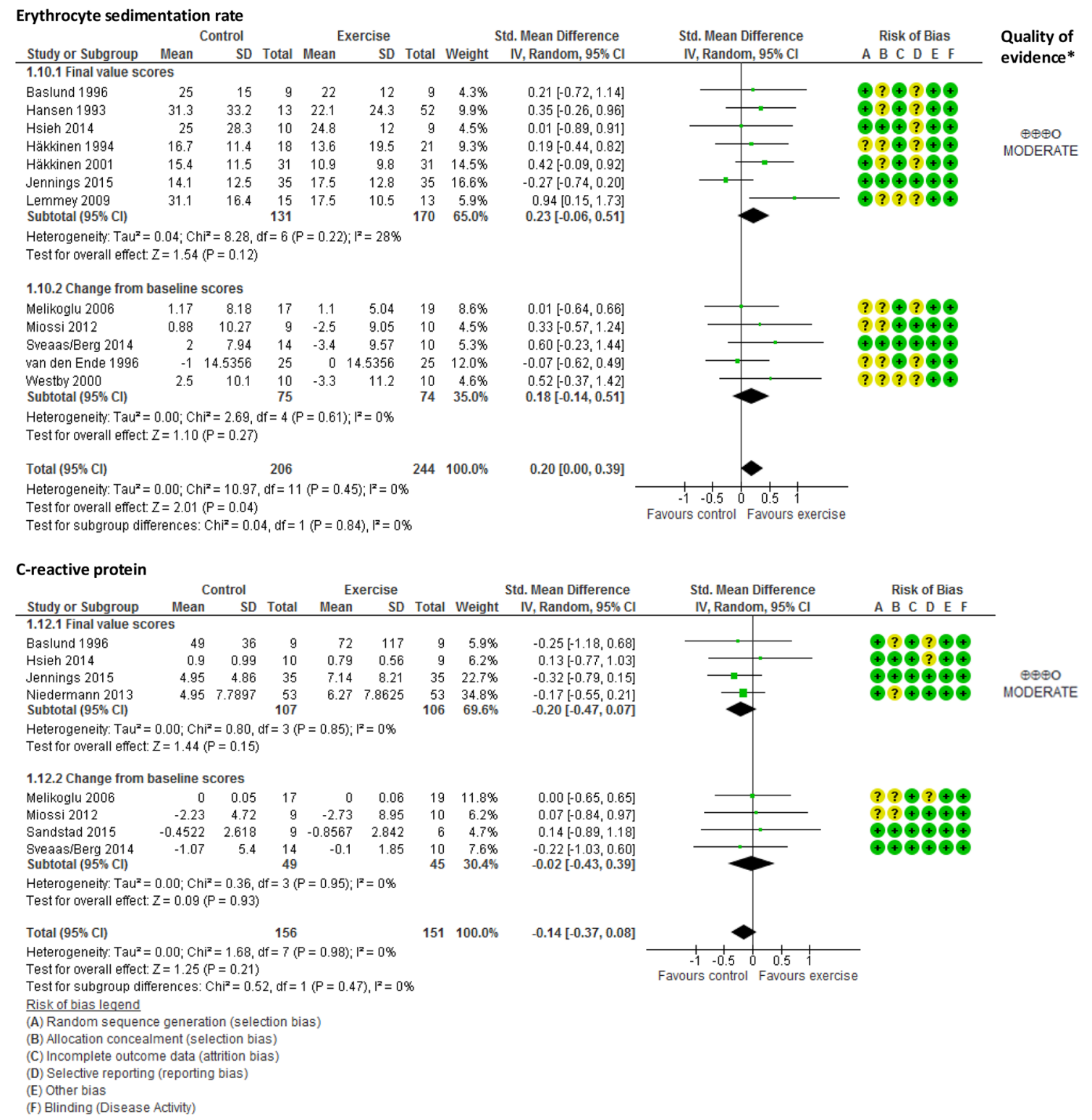

Figure 2 Meta-analyses of effects of exercises on erythrocyte sedimentation rate and $\mathrm{C}$ reactive protein. Values are shown as standardised mean difference with $95 \% \mathrm{Cls}$. ${ }^{*}$ The quality of the evidence was evaluted according to the Grading of RecommendationAssessment, Development and Evaluation approach.

outcome measure of disease activity and was rated as low risk of bias for this item.

For outcomes included in $\geq 10$ studies, the result of Egger's test showed that the mean effect on erythrocyte sedimentation rate (intercept $=2.17, \mathrm{p}=0.05$ ), diagnosis-specific disease activity scores (intercept $=0.22, \mathrm{p}=0.30$ ) and pain (intercept $=-1.15$, $\mathrm{p}=0.09$ ) was not subject to publication bias. The fail-safe number was, however, 7, 10 and 12 for erythrocyte sedimentation rate, diagnosis specific disease activity scores and pain, respectively, thus indicating a risk of publication bias for these outcomes.

\section{Effects}

Inflammation

Twelve RCTs provided data on erythrocyte sedimentation rate, and the results showed moderate quality evidence for a small beneficial effect of exercises (SMD 0.20 (95\% CI 0.00 to 0.39), $\mathrm{p}=0.04$ ) (figure 2 ). There was no between-study heterogeneity $\left(\mathrm{I}^{2}=0 \%\right)$. See online supplementary table S2 for details of the
GRADE. Eight RCTs provided data on C-reactive protein, and there is moderate level of evidence for no effect of exercises (SMD -0.14 (95\% CI -0.37 to 0.08$), p=0.21$ ). Between-study heterogeneity was negligible $\left(\mathrm{I}^{2}=0 \%\right)$. Two RCTs provided data on creatine phosphokinase (a marker of inflammation in muscle tissue) and showed low-quality evidence for a tendency towards a beneficial effect of exercises on this outcome (SMD 1.31 (95\% $\mathrm{CI}-0.20$ to 2.82$), \mathrm{p}=0.09$ ). Between-study heterogeneity was substantial $\left(\mathrm{I}^{2}=62 \%\right)$.

Two RCTs included seven different cytokines and cytokine receptors as outcome measures (shown in online supplementary figure S2). Exercises reduced concentration of interleukin (IL) 17a, IL-18 and tumour necrosis factor alpha receptors, but increased levels of IL-6.

\section{Diagnosis-specific disease activity scores}

Twelve RCTs provided data on diagnosis-specific disease activity scores, and the results showed high-quality evidence for a small 


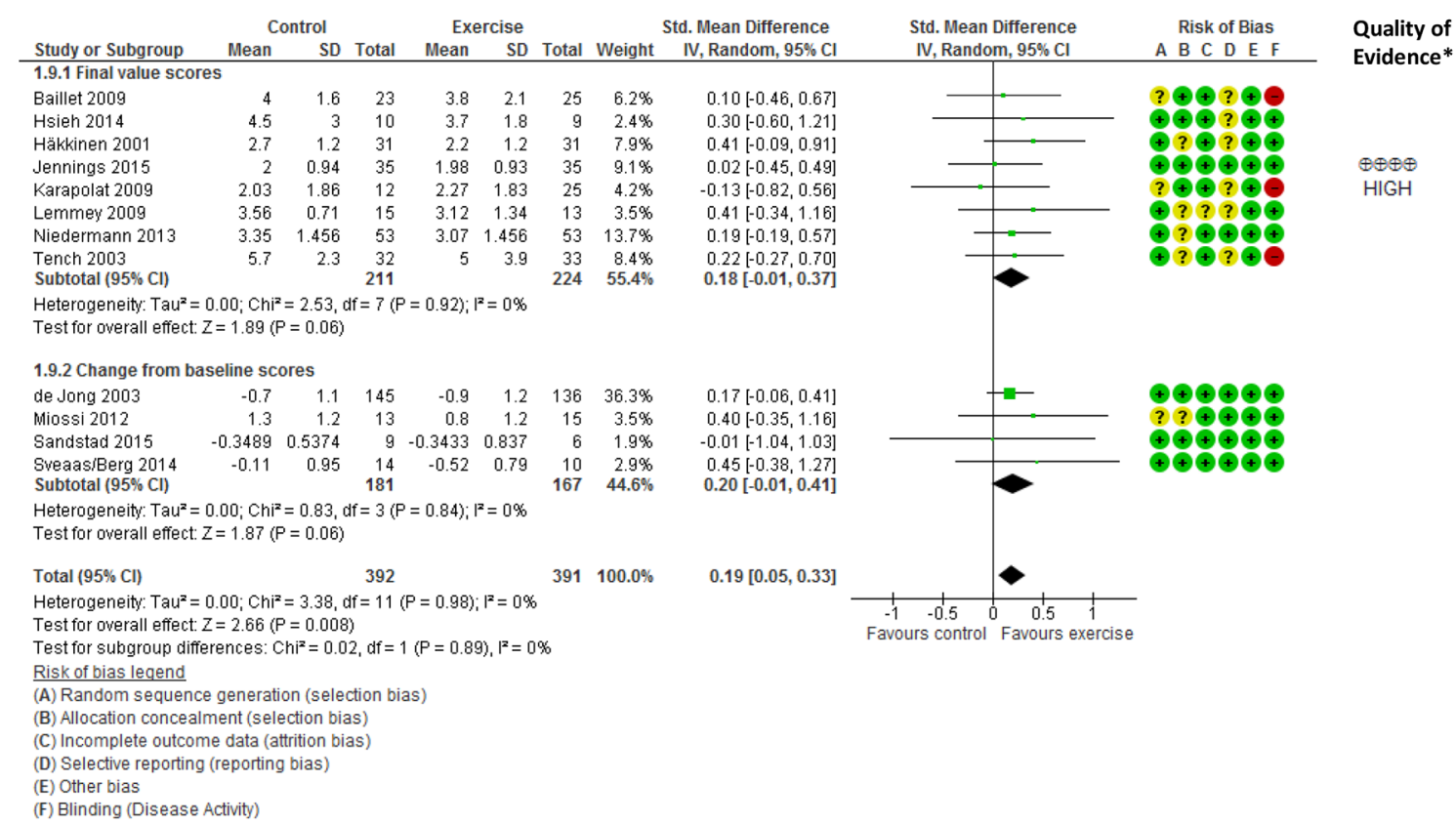

Figure 3 Meta-analysis of effect of exercises on diagnosis-specific disease activity scores. Disease activity was measured with Ankylosing Spondylitis Disease Activity Score, Bath Ankylosing Spondylitis Disease Activity Score, a disease activity score for Rheumatoid Arthritis patients and Systemic Lupus Erythematosus Disease Activity Index. Values are shown as SMD with $95 \% \mathrm{Cls}$. ${ }^{*}$ The quality of the evidence was evaluted according to the Grading of Recommendation Assessment, Development and Evaluation approach.

beneficial effect of exercises (SMD of 0.19 (95\% CI 0.05 to $0.33), \mathrm{p}<0.01$ ) (figure 3). There was no between-study heterogeneity $\left(\mathrm{I}^{2}=0 \%\right)$.

\section{Radiographic joint damage}

Four RCTs provided data on radiographic joint damage in small joints, and the results showed moderate quality evidence for a small beneficial effect of exercises (SMD 0.27 (0.07, 0.46), $\mathrm{p}<0.01$ ) (figure 4). Between-study heterogeneity was negligible $\left(\mathrm{I}^{2}=0 \%\right)$.

\section{Symptoms}

Twelve RCTs provided data on pain, and the results showed moderate quality evidence for a small beneficial effect of exercises (SMD 0.30 (95\% CI 0.13 to 0.47$), \mathrm{p}<0.001$ ) (figure 5). There was no between-study heterogeneity $\left(\mathrm{I}^{2}=0 \%\right)$. Nine RCTs provided data on fatigue, and the results showed moderate quality evidence for a small beneficial effect of exercises (SMD $0.36(95 \%$ CI 0.17 to 0.54$), \mathrm{p}<0.001)$. Between-study heterogeneity was negligible $\left(\mathrm{I}^{2}=0 \%\right)$. Seven RCTs provided data on stiffness, and the results showed moderate quality evidence for a medium beneficial effect of exercises (SMD 0.47 (95\% CI 0.26 to 0.68$), p<0.0001)$. Between-study heterogeneity was negligible $\left(\mathrm{I}^{2}=4 \%\right)$. Five RCTs provided data on number of swollen/ tender joints, and the results showed low quality evidence for a small beneficial effect of exercises ( $\mathrm{SMD}=0.35$ (95\% CI 0.03 to $0.67), \mathrm{p}=0.03$ ) (figure 5 ). There was no between-study heterogeneity $\left(\mathrm{I}^{2}=0 \%\right)$. Four RCTs provided data on joint tenderness,

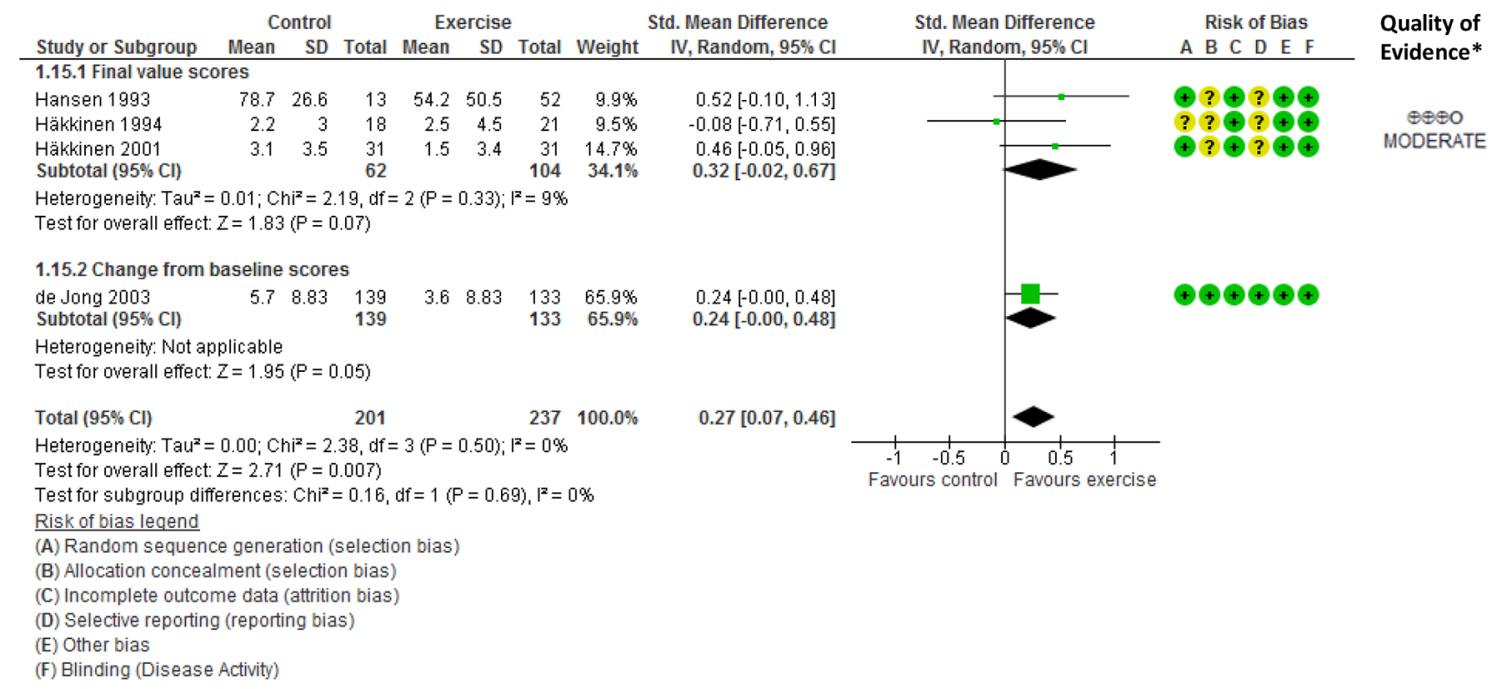

Figure 4 Meta-analysis of effect of exercises on radiographic damage in small joints. Values are shown as SMD with 95\% Cls. *The quality of the evidence was evaluted according to the Grading of Recommendation Assessment, Development and Evaluation approach. 
A pain

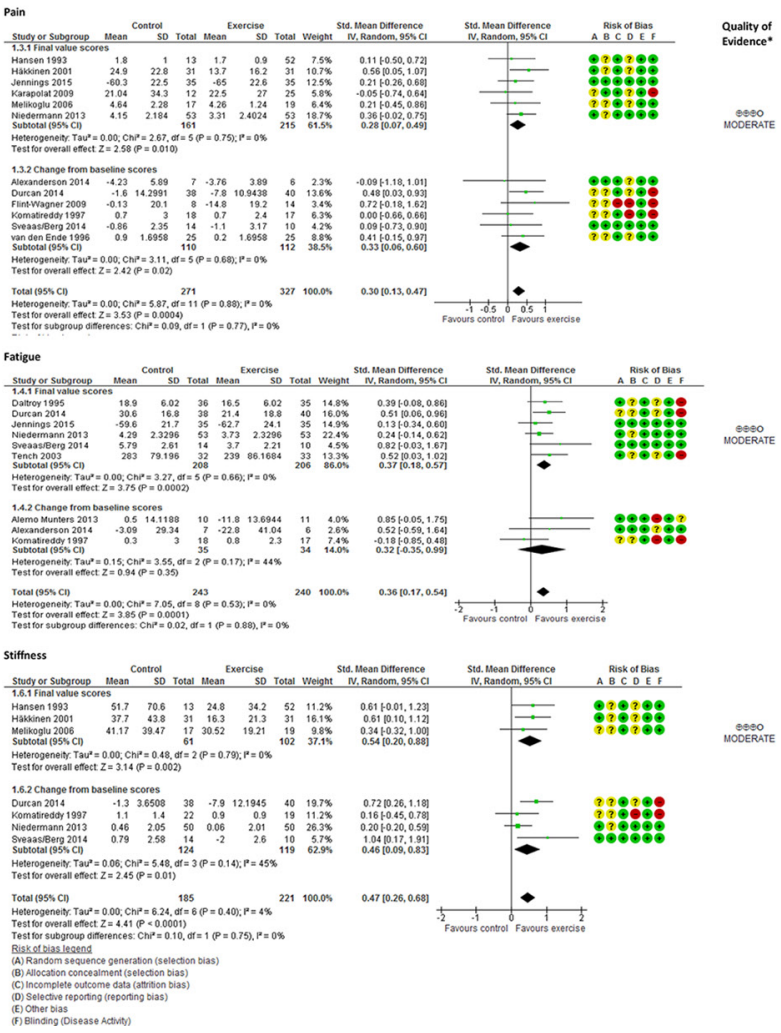

B

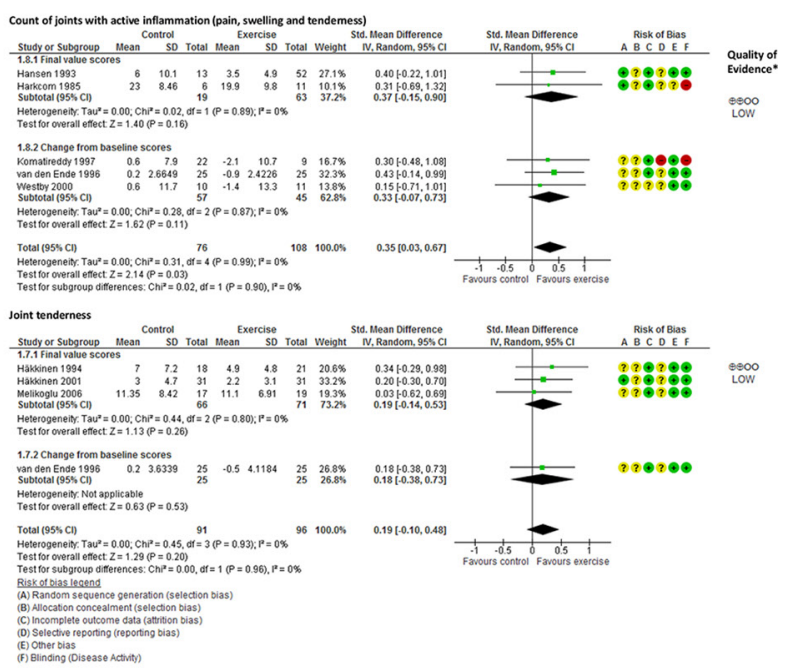

Figure 5 Effect of exercises on symptoms (A) Meta-analyses of effects of exercises on pain, fatigue and stiffness. (B) Meta-analyses of effects of exercises on count of joints with active inflammation (pain, swelling and tenderness and joint tenderness. Values are shown as SMD with $95 \%$ Cls. *The quality of the evidence was evaluted according to the Grading of Recommendation Assessment, Development and Evaluation approach.

and the results showed low quality evidence for no effect of exercises (SMD $0.19(95 \% \mathrm{CI}-0.10$ to 0.48$), \mathrm{p}=0.20)$. There was no between-study heterogeneity $\left(\mathrm{I}^{2}=0 \%\right)$.

\section{Subgroup analyses}

The effect of exercises on erythrocyte sedimentation rate was significant for exercise programmes with $>12$ weeks duration (SMD 0.43 (95\% CI 0.15 to 0.72 )), but not for exercise programmes with a shorter duration (SMD 0.01 (95\% CI -0.25 to 0.27$)$ ), $p=0.03$ for the difference. (see online supplementary table $\mathrm{S} 3$ for details on subgroup analyses). For C-reactive protein, none of the trials had a duration $>12$ weeks. Except for erythrocyte sedimentation rate, the subgroup analyses on duration revealed no differences on the other outcomes. The subgroup analyses on exercise mode revealed no differences. Finally, no differences between the supervised and unsupervised exercise programmes on any outcomes were found. The same results were seen when using meta-regression to explore subgroup differences (relevant for meta-analyses with $>10$ included trials, ie, erythrocyte sedimentation rate, diagnosis specific disease activity scores and pain).

\section{Adverse events and side effects}

Only six trials included the reporting of adverse events/side effects in their articles. ${ }^{26} 2730374552$ No adverse events were reported, but one trial reported that patients in the exercise group experienced short-term muscle soreness. ${ }^{26}$

\section{DISCUSSION}

Overall, in this systematic review, including 26 trials and 1286 patients with IRDs, the results suggest beneficial effect of exercises on disease activity in terms of inflammation, joint damage and symptoms. There was moderate to high quality evidence for small beneficial effects of exercises on diagnosis-specific disease activity scores, joint damage and erythrocyte sedimentation rate. Furthermore, there was moderate to low quality evidence for small to medium beneficial effects of exercises on symptoms.

According to current treatment recommendations, exercise should complement pharmacological treatment in patients with IRDs. ${ }^{54}$ This meta-analysis showed that exercise may have beneficial effects on both inflammation and symptoms. Even though pharmacological treatment has been substantially improved in recent years, comprehensive pharmacological treatment is only recommended if the symptoms are caused by inflammation. ${ }^{545}$ Hence, in cases where the symptoms are not caused by current inflammation and for patients not responding to pharmacological treatment, cardiorespiratory and strength exercises are especially important as treatment alternatives. Although this review showed small to medium effect sizes, the studies consistently conclude with reduced disease activity and symptoms following exercise interventions, underlining the important role of exercises as treatment for patients with IRDs. In contrast to empirical beliefs that exercises might cause flare ups in disease activity, the beneficial effects on inflammation and symptoms ensure that exercises are safe for patients with IRDs. Thus, patients with IRDs can take advantage of the numerous health effects of cardiorespiratory and strength exercises.

In addition to the disease-modifying effect, cardiorespiratory and strength exercises also have the potential to prevent comorbidity in patients with IRDs. There is growing evidence that these patients have an increased risk of CVD, ${ }^{56}$ and cardiorespiratory and strength exercises are recognised as cornerstones in the prevention of CVD. ${ }^{87}$ However, exercises to maintain flexibility and mobility, the exercise modalities traditionally 
recommended for patients with IRDs, ${ }^{6}$ do not protect against CVD. ${ }^{8}$ Thus, in light of the high risk of CVD associated with IRDs, the beneficial effects of cardiorespiratory and strength exercises should be exploited to optimise the treatment for patients with IRDs.

The results of the current review are in accordance with two other meta-analyses showing that cardiorespiratory and strength exercises had beneficial effects on inflammation, pain and joint tenderness in patients with rheumatoid arthritis. ${ }^{18}{ }^{19}$ To the best of our knowledge, this is the first meta-analysis summarising the effects of cardiorespiratory and strength exercises in patients with different IRD diagnoses, and the results support a hypothesis that exercises can counteract inflammation and clinical symptoms. ${ }^{14-17}$ Even if the exercise programmes included in this meta-analysis were in accordance with the ACSM's exercise recommendations, ${ }^{8}$ the doses were relatively low in many of the studies. For instance, only four trials described a vigorous intensity level. Since the physiological responses to exercise, including the anti-inflammatory effect, ${ }^{17}$ are dependent on dosage, it may be hypothesised that higher doses could possibly have provided larger effect sizes. Further research is needed to gain more insight into the dose-response relationship for the effect of exercises on disease activity.

The exploratory subgroup analyses showed that only exercise programmes with a long duration were effective in reducing erythrocyte sedimentation rate, whereas no studies provided data on effect of long-term exercise on $\mathrm{C}$ reactive protein. The anti-inflammatory benefit of exercises is described to be an acute effect of each bout of exercise, ${ }^{9}$ and the findings in this meta-analysis indicate that exercise may suppress inflammation over time. Furthermore, subgroup analyses suggested that only cardiorespiratory exercises and a combination of cardiorespiratory and strength exercises were beneficial in reducing fatigue. Except for this outcome, there were no significant differences between the different exercise modes, and more research is therefore needed to conclude on the most optimal exercise mode for reducing inflammation and symptoms in patients with IRDs.

Strengths of this review are the comprehensive literature search, the large number of trials included, the grading of evidence and the statistical pooling in a meta-analysis. In addition, the assessment of direct measures of disease activity strengthens the validity of the results. Blinding of the participants in exercise interventions is not possible, and if the results had been based solely on patient-reported outcomes, a possible placebo effect could not have been ruled out.

A limitation of this review is that follow-up studies were not included; hence, the long-term effects of exercises on disease activity are still unknown. Furthermore, adherence to the exercise interventions either as number of completed exercise sessions or as actual dosage of exercises was not explored. Moreover, only six different diagnoses were included with the majority of the patients having rheumatoid arthritis and axial spondyloarthritis, and therefore, the generalisation of the results to other IRD diagnoses should be done with caution. Based on an explorative approach, all types of disease activity measures were analysed in this systematic review, but only outcomes applied in two or more trials were pooled, which may have led to a reporting bias. In addition, as the fail-safe numbers indicated a possible risk of publication bias, inflated estimates of intervention effects cannot be ruled out.

Furthermore, adverse events may have been under-reported, as only six trials addressed this issue. However, the risk of minor injuries associated with exercise is probably considered of minor interest, as this risk must be regarded as similar for IRD patients as for healthy adults. An important expression of safety for IRD patients is, however, the consistent result of no worsening of disease activity related to exercise shown in this meta-analysis.

There is currently a focus on reducing years lived with disability globally, as these rates are declining much more slowly than mortality rates. ${ }^{58}$ This meta-analysis indicates that cardiorespiratory and strength exercises can reduce the burden of disease in patients with IRDs. As these patients often live many years with disability, it is of utmost importance to exploit the disease-modifying effects of cardiorespiratory and strength exercises.

\section{CONCLUSION}

This meta-analysis showed that cardiorespiratory and strength exercises, dosed according to recommendations for improving physical fitness, have beneficial effects on disease activity in terms of inflammation, joint damage and symptoms in patients with IRDs. Therefore, cardiorespiratory and strength exercises can be considered as safe and effective therapeutic tools. Future studies are, however, needed to explore the most optimal exercise mode and dose. Furthermore, future reviews should aim at identifying potential moderating variables of exercises thorough meta-analyses based on individual patient data, as this is the preferred method of summarising RCTs.

\section{What are the new findings?}

- Cardiorespiratory and strength exercises, dosed according to recommendations for improving physical fitness, may have beneficial effects on disease activity in terms of inflammation, joint damage and symptoms, and should therefore be included as a part of the treatment programme for patients with inflammatory rheumatic diseases (IRDs).

- Cardiorespiratory and strength exercises are safe and beneficial for patients with IRDs, and this finding is contrary to empirical beliefs that exercises might cause flare ups in disease activity.

- By engaging in cardiorespiratory and strength exercises, patients with IRDs will be able to take advantage of the numerous health effects of increased physical fitness, such as better cardiovascular health.

Correction notice This paper has been amended since it was published Online First. Owing to a scripting error, some of the publisher names in the references were replaced with 'BMJ Publishing Group'. This only affected the full text version, not the PDF. We have since corrected these errors and the correct publishers have beeninserted into the references.

Contributors SHS, KBH and HD designed the study. All authors acquired the data. SHS and GS coded and analysed the data. All authors drafted the manuscript and approved the final version to be published.

Funding This work was supported by The Norwegian Fund for Post-Graduate Training in Physiotherapy.

Competing interests None declared.

Provenance and peer review Not commissioned; externally peer reviewed.

(C) Article author(s) (or their employer(s) unless otherwise stated in the text of the article) 2017. All rights reserved. No commercial use is permitted unless otherwise expressly granted.

\section{REFERENCES}

1 Goldblatt F, O'Neill SG. Clinical aspects of autoimmune rheumatic diseases. Lancet 2013;382:797-808.

2 Atzeni F, Masala IF, Salaffi F, et al. Pain in systemic inflammatory rheumatic diseases. Best Pract Res Clin Rheumatol 2015;29:42-52.

3 Mason JC, Libby P. Cardiovascular disease in patients with chronic inflammation: mechanisms underlying premature cardiovascular events in rheumatologic conditions. Eur Heart J 2015;36:482-9.

4 Smolen JS. Treat-to-target as an approach in inflammatory arthritis. Curr Opin Rheumatol 2016;28:297-302. 
5 Smolen JS, Braun J, Dougados M, et al. Treating spondyloarthritis, including ankylosing spondylitis and psoriatic arthritis, to target: recommendations of an international task force. Ann Rheum Dis 2014;73:6-16.

6 Dagfinrud H, Halvorsen S, Vøllestad NK, et al. Exercise programs in trials for patients with ankylosing spondylitis: do they really have the potential for effectiveness? Arthritis Care Res 2011;63:597-603.

7 Caspersen CJ, Powell KE, Christenson GM. Physical activity, exercise, and physical fitness: definitions and distinctions for health-related research. Public Health Rep 1985;100:126-31.

8 Garber CE, Blissmer B, Deschenes MR, et al; American College of Sports Medicine. American college of sports medicine position stand. quantity and quality of exercise for developing and maintaining cardiorespiratory, musculoskeletal, and neuromotor fitness in apparently healthy adults: guidance for prescribing exercise. Med Sci Sports Exerc 2011;43:1334-59.

9 Walsh NP, Gleeson M, Shephard RJ, et al. Position statement. part one: immune function and exercise. Exerc Immunol Rev 2011;17:6-63.

10 Fedewa MV, Hathaway ED, Ward-Ritacco CL. Effect of exercise training on C-reactive protein: a systematic review and meta-analysis of randomised and non-randomised controlled trials. Br J Sports Med 2016

11 Metsios GS, Stavropoulos-Kalinoglou A, Panoulas VF, et al. Association of physical inactivity with increased cardiovascular risk in patients with rheumatoid arthritis. Eur J Cardiovasc Prev Rehabil 2009;16:188-94.

12 Halvorsen S, Vøllestad NK, Provan SA, et al. Cardiorespiratory fitness and cardiovascular risk in patients with ankylosing spondylitis: a Cross-Sectional comparative study. Arthritis Care Res 2013;65:969-76.

13 Plasqui G, Boonen A, Geusens P, et al. Physical activity and body composition in patients with ankylosing spondylitis. Arthritis Care Res 2012;64:101-7.

14 Benatti FB, Pedersen BK. Exercise as an anti-inflammatory therapy for rheumatic diseases-myokine regulation. Nat Rev Rheumatol 2015;11:86-97.

15 Perandini LA, de Sá-Pinto AL, Roschel $\mathrm{H}$, et al. Exercise as a therapeutic tool to counteract inflammation and clinical symptoms in autoimmune rheumatic diseases. Autoimmun Rev 2012;12:218-24.

16 Ploeger HE, Takken T, de Greef MH, et al. The effects of acute and chronic exercise on inflammatory markers in children and adults with a chronic inflammatory disease: a systematic review. Exerc Immunol Rev 2009;15:6-41.

17 Beavers KM, Brinkley TE, Nicklas BJ. Effect of exercise training on chronic inflammation. Clin Chim Acta 2010;411:785-93.

18 Baillet A, Vaillant M, Guinot M, et al. Efficacy of resistance exercises in rheumatoid arthritis: meta-analysis of randomized controlled trials. Rheumatology 2012;51:519-27.

19 Baillet A, Zeboulon N, Gossec L, et al. Efficacy of cardiorespiratory aerobic exercise in rheumatoid arthritis: meta-analysis of randomized controlled trials. Arthritis Care Res 2010;62:984-92

20 Wan X, Wang W, Liu J, et al. Estimating the sample mean and standard deviation from the sample size, median, range and/or interquartile range. BMC Med Res Methodol 2014;14:135

21 Comprehensive Meta-Analysis version 3. Englewood, NJ: Biostat, 2014. Computer softeware available from http://www.comprehensive.com.

22 Higgins J, Altman D. Assessing risk of bias in included studies. In: Higgins JPT, Green $S$, eds. Cochrane handbook for systematic reviews of interventions. Chichester: wileyblackwell, 2008:187-235.

23 Sterne J, N.; Moher D. Addressing reporting biases. In: Higgins JPT, Green S, eds. Cochrane handbook for systematic reviews of interventions. Chichester: wileyblackwell, 2008:297-325.

24 Cohen J. Statistical power analysis for the behavioral sciences: Hillsdale, NJ Laurence Erlbaum, 1988

25 Deeks JJ, Altman DG. Analysing data and undertaking meta-analyses. In: Higgins JPT, Green S, eds. Cochrane handbook of systematic reviews of interventions. Chichester: wiley-blackwell, 2008:243-93.

26 Alexanderson H, Munters LA, Dastmalchi M, et al. Resistive home exercise in patients with recent-onset polymyositis and dermatomyositis - a randomized controlled single-blinded study with a 2-year followup. I Rheumatol 2014:41:1124-32.

27 Miossi R, Benatti FB, Lúciade de Sá Pinto A, et al. Using exercise training to counterbalance chronotropic incompetence and delayed heart rate recovery in systemic lupus erythematosus: a randomized trial. Arthritis Care Res 2012;64:1159-66.

28 Niedermann K, Sidelnikov E, Muggli C, et al. Effect of cardiovascular training on fitness and perceived disease activity in people with ankylosing spondylitis. Arthritis Care Res 2013:65:1844-52.

29 Sandstad J, Stensvold D, Hoff M, et al. The effects of high intensity interval training in women with rheumatic disease: a pilot study. Eur J Appl Physiol 2015;115:2081-9.

30 Sveaas SH, Berg IJ, Provan SA, et al. Efficacy of high intensity exercise on disease activity and cardiovascular risk in active axial spondyloarthritis: a randomized controlled pilot study. PLoS One 2014;9:e108688.

31 Hansen TM, Hansen G, Langgaard AM, et al. Longterm physical training in rheumatoid arthritis. A randomized trial with different training programs and blinded observers. Scand I Rheumatol 1993;22:107-12.

32 Harkcom TM, Lampman RM, Banwell BF, et al. Therapeutic value of graded aerobic exercise training in rheumatoid arthritis. Arthritis \& Rheumatism 1985;28:32-9.
33 Tench $\mathrm{CM}$, et al. Fatigue in systemic lupus erythematosus: a randomized controlled trial of exercise. Rheumatology 2003;42:1050-4.

34 Daltroy LH, Robb-Nicholson C, Iversen MD, et al. Effectiveness of minimally supervised home aerobic training in patients with systemic rheumatic disease. $B$ J Rheumatol 1995;34:1064-9.

35 de Jong Z, Munneke $M$, Zwinderman AH, et al. Long term high intensity exercise and damage of small joints in rheumatoid arthritis. Ann Rheum Dis 2004;63:1399-405.

36 Baslund B, Lyngberg K, Andersen V, et al. Effect of 8 wk of bicycle training on the immune system of patients with rheumatoid arthritis. J Appl Physiol 1993:75:1691-5.

37 Baillet A, Payraud E, Niderprim VA, et al. A dynamic exercise programme to improve patients' disability in rheumatoid arthritis: a prospective randomized controlled trial. Rheumatology 2009:48:410-5.

38 de Jong Z, Munneke M, Zwinderman AH, et al. Is a long-term high-intensity exercise program effective and safe in patients with rheumatoid arthritis? results of a randomized controlled trial. Arthritis Rheum 2003;48:2415-24.

39 Durcan L, Wilson F, Cunnane $G$. The effect of exercise on sleep and fatigue in rheumatoid arthritis: a randomized controlled study. J Rheumatol 2014;41:1966-73.

40 Flint-Wagner HG, Lisse J, Lohman TG, et al. Assessment of a sixteen-week training program on strength, pain, and function in rheumatoid arthritis patients. J Clin Rheumatol 2009:15:165-71.

41 Häkkinen A, Häkkinen K, Hannonen P. Effects of strength training on neuromuscular function and disease activity in patients with recent-onset inflammatory arthritis. Scand I Rheumatol 1994;23:237-42.

42 Häkkinen A, Sokka T, Kotaniemi A, et al. A randomized two-year study of the effects of dynamic strength training on muscle strength, disease activity, functional capacity, and bone mineral density in early rheumatoid arthritis. Arthritis Rheum 2001:44:515-22.

43 Häkkinen A, Sokka T, Lietsalmi A-M, et al. Effects of dynamic strength training on physical function, valpar 9 work sample test, and working capacity in patients with recent-onset rheumatoid arthritis. Arthritis Care Res 2003:49:71-7.

44 Komatireddy GR, Leitch RW, Cella K, et al. Efficacy of low load resistive muscle training in patients with rheumatoid arthritis functional class II and III. J Rheumatol 1997:24:1531-9.

45 Lemmey AB, Marcora SM, Chester K, et al. Effects of high-intensity resistance training in patients with rheumatoid arthritis: a randomized controlled trial. Arthritis \& Rheumatism 2009;61:1726-34.

46 Melikoglu MA, Karatay S, Senel K, et al. Association between dynamic exercise therapy and IGF- 1 and IGFBP-3 concentrations in the patients with rheumatoid arthritis. Rheumatol Int 2006:26:309-13.

47 van den Ende $\mathrm{CH}$, Hazes JM, le Cessie S, et al. Comparison of high and low intensity training in well controlled rheumatoid arthritis. results of a randomised clinical trial. Ann Rheum Dis 1996;55:798-805.

48 Westby MD, Wade JP, Rangno KK, et al. A randomized controlled trial to evaluate the effectiveness of an exercise program in women with rheumatoid arthritis taking low dose prednisone... J Rheumatol 2000;27:1674-80.

49 Hsieh L-F, Chuang C-C, Tseng C-S, et al. Combined home exercise is more effective than Range-of-Motion home exercise in patients with ankylosing spondylitis: a randomized controlled trial. Biomed Res Int 2014:2014:1-9.

50 Jennings $F$, Oliveira HA, de Souza MC, et al. Effects of aerobic training in patients with ankylosing spondylitis. J Rheumato/ 2015:42:2347-53.

51 Karapolat $\mathrm{H}$, Eyigor S, Zoghi M, et al. Are swimming or aerobic exercise better than conventional exercise in ankylosing spondylitis patients? a randomized controlled study. Eur J Phys Rehab Med 2009;45:449-57.

52 Alemo Munters L, Dastmalchi M, Andgren V, et al. Improvement in health and possible reduction in disease activity using endurance exercise in patients with established polymyositis and dermatomyositis: a multicenter randomized controlled trial with a 1-year open extension followup. Arthritis Care Res 2013:65:1959-68.

53 Wiesinger $\mathrm{G}$, Quittan $\mathrm{M}$, Graninger $\mathrm{M}$, et al. Benefit of 6 months long-term physical training in polymyositis/dermatomyositis patients. Rheumatology 1998:37:1338-42.

54 Ward MM, Deodhar A, AkI EA, et al. American college of rheumatology/Spondylitis association of america/Spondyloarthritis research and treatment network 2015 recommendations for the treatment of ankylosing spondylitis and nonradiographic axial spondyloarthritis. Arthritis Rheumatol 2016;68:282-98.

55 Smolen JS, Landewé R, Breedveld FC, et al. EULAR recommendations for the management of rheumatoid arthritis with synthetic and biological disease-modifying antirheumatic drugs: 2013 update. Ann Rheum Dis 2014;73:492-509.

56 Hollan I, Meroni PL, Ahearn JM, et al. Cardiovascular disease in autoimmune rheumatic diseases. Autoimmun Rev 2013;12:1004-15.

57 Barlow CE, DeFina LF, Radford NB, et al. Cardiorespiratory fitness and Long-Term survival in "Low-Risk" Adults. J Am Heart Assoc 2012;1:e001354.

58 Global Burden of Disease Study 2013 Collaborators. Global, regional, and national incidence, prevalence, and years lived with disability for 301 acute and chronic diseases and injuries in 188 countries, 1990-2013: a systematic analysis for the global burden of disease study 2013. Lancet 2015;386:743-800. 